Research Article

Olga Andreeva*, Valentina Abramenko, and Valentina Malashchuk

\title{
11-year dynamics of coronal hole and sunspot areas
}

https://doi.org/10.1515/astro-2022-0005

Received Nov 12, 2021; accepted Jan 03, 2022

\begin{abstract}
The paper presents study the 11-year dynamics of solar activity on the basis of new observational material on coronal holes (CHs) and sunspots in the period from May 13, 2010 to May 13, 2021. We used the Heliophysics Event Knowledgebase (HEK) to obtain information on CHs areas. For 11 years of observations, we processed about 18000 CHs. Slightly more than 8000 are polar, the rest are nonpolar CHs. The statistical volume of the presented material is quite extensive and gives grounds for the study of the dynamics of different types of CHs during the cycle. Our research has shown: in the 24th solar activity cycle, the South led for polar CHs and the North led for nonpolar ones. We established a relationship between the number and area of $\mathrm{CHs}$ and the phase of the solar cycle. The number and daily total area of polar CHs increases at the minima of solar activity and decreases at the maximum of the cycle. This is consistent with the general concept of polar CHs as the main source of the solar dipole magnetic field. An asymmetry in both the number and areas of polar coronal holes in the northern and southern hemispheres is observed. It is shown that the areas of nonpolar CHs change quasi-synchronously with sunspot activity, which suggests a physical connection between these two phenomena.
\end{abstract}

Keywords: Sun, solar cycle, solar activity, coronal holes, sunspots

\section{Introduction}

The solar cycle is a dynamo process in which poloidal magnetic field is twisted by differential rotation into a toroidal field and then that toroidal field is transformed by cyclonic convection back into a poloidal field of the opposite sign (Parker 1955; Dikpati and Charbonneau 1999). The original polarity is then recovered after the second solar cycle. The 11-year cycle is characterized by periodic changes in measures of solar activity such as the number of sunspots, coronal holes, and others. During a solar maximum, the sunspot number reaches a maximum, coronal holes extend to low latitudes and are diminished in polar regions, coronal cavities and prominences migrate toward the pole ( $\mathrm{Li}$ et al. 2008; Shimojo 2013; Karna 2015). At the same time, the polar open magnetic field weakens, decreases through zero, and then emerges again with the opposite polarity.

Regions on the Sun with open magnetic fields are sources of the fast solar wind, and thus play a crucial role

Corresponding Author: Olga Andreeva: Crimean Astrophysical Observatory, Russian Academy of Sciences, Nauchny, 298409, Russia; Email: olga.a7@gmail.com

Valentina Abrameko: Crimean Astrophysical Observatory, Russian Academy of Sciences, Nauchny, 298409, Russia

Valentina Malashchuk: Crimean Astrophysical Observatory, Russian Academy of Sciences, Nauchny, 298409, Russia in Heliophysics as a main driver of geomagnetic activity. Key structures indicative of open magnetic fields are coronal holes, which are large regions of the corona that are unipolar, low density regions that appear to be dark when observed both on the solar disk and above the solar limb in EUV observations (Cranmer 2009). During solar maximum the area of the polar coronal holes diminishes while the area of equatorial coronal holes increases.

In order to statistically investigate the relationship between coronal holes and sunspots during the solar cycle, we study the cyclic variations in their areas. The study of variations in the areas of polar and nonpolar coronal holes was carried out earlier. For example, Fujiki et al. (2016) investigated the long-term trend of the distribution of coronal holes in the Sun from 1975 to 2014. Lowder et al. (2017) detailed examination of the latitude dependence of coronal hole properties over cycles 23 and 24. Our paper focuses on an analysis of the 11-year dynamics of the two types of coronal holes in the N- and S- hemispheres based on SDO epoch data observation.

\section{Data, method and statistics}

Our study used extreme ultraviolet images of the Sun in the Fe XII line (19.3 nm), obtained with the AIA/SDO. To localize coronal holes and determine their areas, we used 

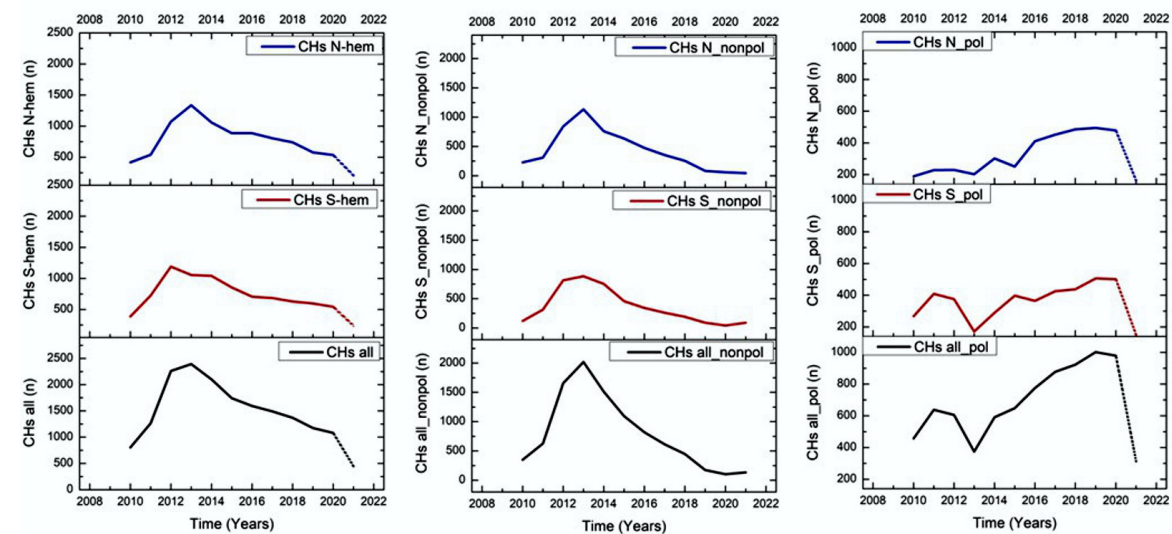

Figure 1. Change in the number of coronal holes in the period from May 13, 2010 to May 13, 2021. Panels from left to right:all, nonpolar, and polar $\mathrm{CHs}$. The plots for the $\mathrm{N}$ and $\mathrm{S}$ hemispheres and for the entire visible surface of the Sun are located in each panel from top to bottom, respectively.

the Heliophysics Event Knowledgebase (HEK) (Hurlburt et al. 2012), which is available at http://www.lmsal.com/hek/ hek_isolsearch.html. Information on coronal holes was extracted with the Spatial Possibilistic Clustering Algorithm (SPoCA) which was described in detail by Barra et al. (2009) and Verbeeck et al. (2014). The software performs the segmentation of the Sun images on active regions, quiet Sun, and $\mathrm{CHs}$ and make records into the HEK catalogs every four hours. These databases made it possible to obtain an array of daily total CHs areas for the period May 13, 2010-May 13, 2021. The area measured in square mega meters. For 11 years of observations, we processed about 18000 CHs. Slightly more than 8000 are polar, the rest are nonpolar CHs. The statistical volume of the presented material is quite extensive and gives grounds for the study of the dynamics of different types of $\mathrm{CHs}$ during the cycle. In (Andreeva and Malashchuk 2020) we explained our separation of CHs into polar and nonpolar. Below we briefly describe it. Statistics on the number of all types of coronal holes during the study period are presented graphicaly in Figure 1. It can be seen that the maximum, determined by the total number of $\mathrm{CHs}$ (lower left panel plot) is due to two maxima: first in the South, then in the North (middle and upper plots). At the same time, we show that the number of holes crossing the equator was maximal in 2012, the first maximum of the 24 th cycle. We can also note that the maximum of the total number of CHs (lower left panel plot) is determined by the maximum number of all nonpolar $\mathrm{CHs}$ (lower middle panel plot). Their number was significantly higher in 2011-2015. At the same time, especially in 2013, the total number of polar CHs (lower graph in the right panel) was minimal. The right panel shows that the South led by the number of polar CHs during the growth phase, while the number of polar CHs in both hemispheres had maximum values during the decline phase and the $24-25$ cycle minimum. The dip in the right panel is artificial, as we are not looking at data for the entire year 2021, but only before May 13 .

The physical conclusion: nonpolar holes repeat the cycle. Polar holes behave in accordance with the general dipole of the solar magnetic field. Their physical nature is quite different and nonpolar CHs must most likely be associated with the sunspots activity. We drew this conclusion from the number of coronal holes. Now we turn to the analysis by the areas of coronal holes (Sch).

\section{Relationship between the coronal holes and sunspots during 11-year}

During the study, we analyzed $17717 \mathrm{CHs}$, of which less than $7 \%$ crossed the equator. We assumed that the area of $\mathrm{CHs}$ crossing the equator is divided into 2 parts, and each of them refers to the corresponding hemisphere, depending on its location. In our opinion, we did not make a big mistake, because there were quite few such CHs. Given this assumption, $9064 \mathrm{CHs}$ were observed in the $\mathrm{N}$ hemisphere and $8653 \mathrm{CHs}$ in the $\mathrm{S}$ hemisphere. As in our earlier works, here we study two groups of $\mathrm{CHs}$ (Figure 2): polar (CH_pol) and nonpolar (CH_nonpol). $\mathrm{CH} \_$pol begin at the poles, i.e., at 90 degrees latitude, and descend, depending on their development and cycle phase, sometimes to middle and even low latitudes. $\mathrm{CH} \_n o n p o l$ are mid- and low-latitude $\mathrm{CHs}$ that are not associated with the pole. The division of $\mathrm{CHs}$ into 2 groups, which we adopted in our study, is described in detail in (Andreeva and Malashchuk 2020). 


\subsection{Dynamics of coronal holes area}

As a result of the processing of the array of $\mathrm{CHs}$ areas extracted from the EUV images by the SPoCA, we obtained the temporal variations of the daily total $\mathrm{CHs}$ area (Sch) for the entire visible surface of the solar disk and separately for both hemispheres (Figure 3). In all three plots of Figure 3, the gray lines are Sch and the red curves are the 6-monthly smoothed values of the corresponding components. Visible quasi-periodic oscillations on the middle and bottom plots for Sch are an artifact caused by the tilt of the sun's rotation axis during the year. It can be seen that the $\mathrm{N}$ and $\mathrm{S}$ hemispheres operated differently during the period under study. The South led by the maximum area of $\mathrm{CHs}$ in the growth phase of solar activity in cycle 24 (2011-2012). In the second maxima (2014) and on the decline branch, the North began to dominate.

Figure 4 from top to bottom show changes in the daily total area (gray lines) for all, polar and nonpolar CHs on the visible surface of the solar disk. The color curves are the CHs areas smoothed over 6 months. Two maxima of the polar CHs area are observed, which fall on 2011-2012 and 2015 - phases of growth and decline of solar activity. After the decline phase and at the minimum of cycle 2425 , the total areas of polar $\mathrm{CHs}$ approach an average value of about $14^{\star} 10^{4} \mathrm{Mm}^{2}$. The total areas of nonpolar CHs in the beginning of the period under study have low values, practically disappear during the decline and at $\min 24-25$ cycles and appear at the growth branch of cycle 25 . The maximum areas of nonpolar $\mathrm{CHs}$ are consistent with the second phase of the maximum of cycle in 2013-2014.
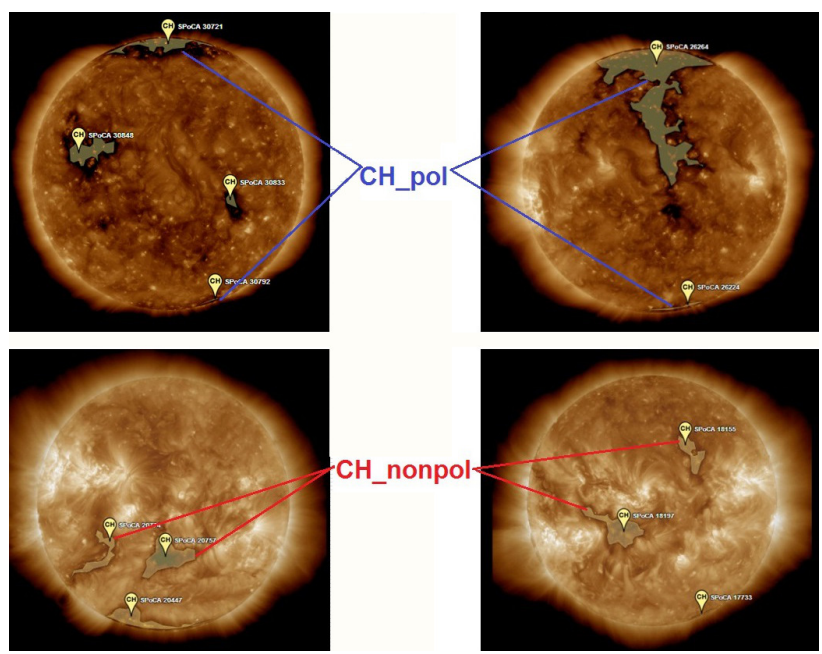

Figure 2. Two types of coronal holes. The areas on the solar disk marked with $\mathrm{CH}$ are $\mathrm{CHs}$ extracted from the EUV image with SPoCA. $\mathrm{CH}_{\text {_pol }}$ - polar and $\mathrm{CH}$ _nonpol - nonpolar or isolated $\mathrm{CHs}$. Bright formations on the solar disk are active regions.

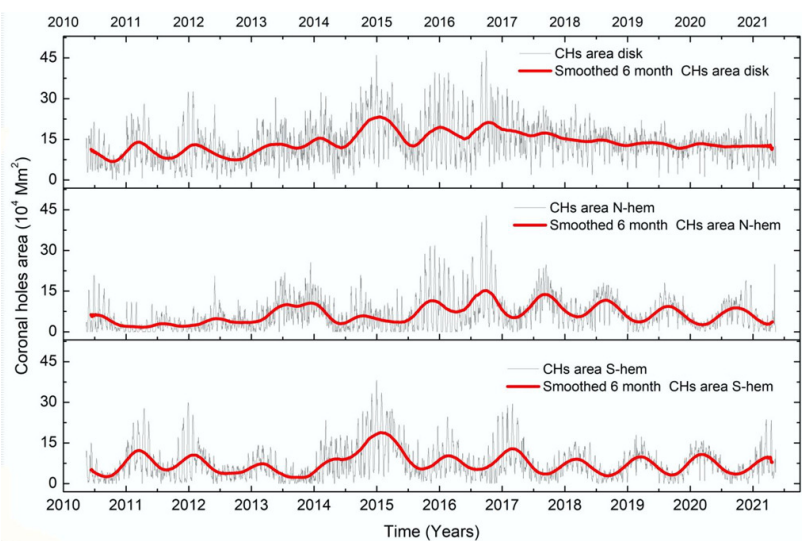

Figure 3. Dynamics of the areas of all $\mathrm{CHs}$ on the visible surface of the solar disk, in $\mathrm{N}$ and $\mathrm{S}$ hemispheres (from top to bottom) in the period May 13, 2010 - May 13, 2021.

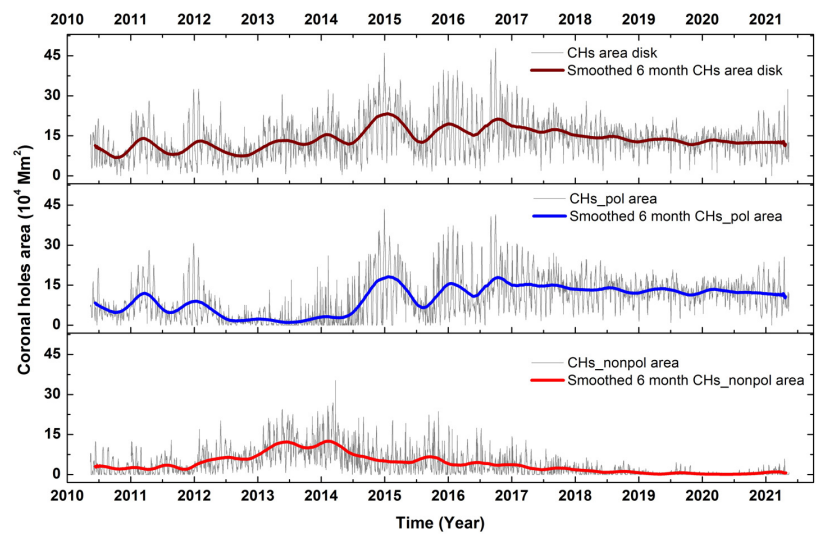

Figure 4. Dynamics of the areas of all, polar and nonpolar CHs (from top to bottom) on the visible surface of the solar disk in period from May 13, 2010 to May 13, 2021.

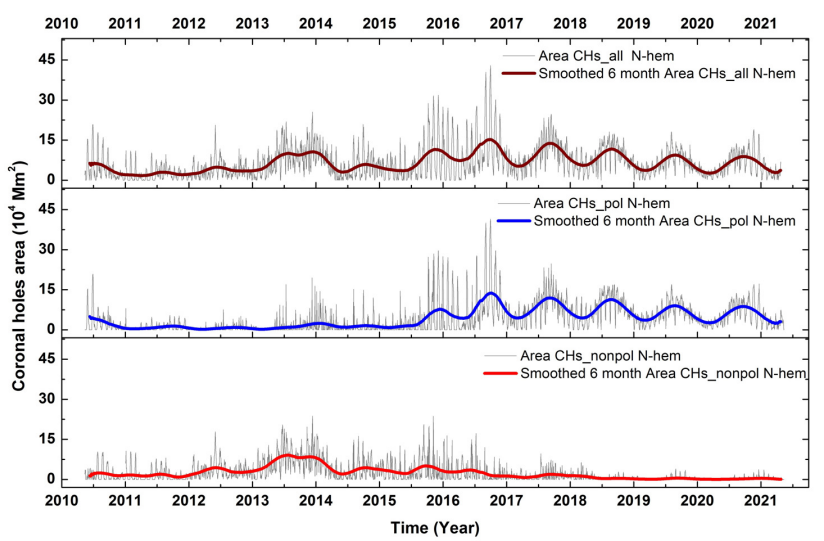

Figure 5. Dynamics of the areas of all, polar and nonpolar CHs (from top to bottom) in the N-hemisphere in the period May 13, 2010 to May 13, 2021. 


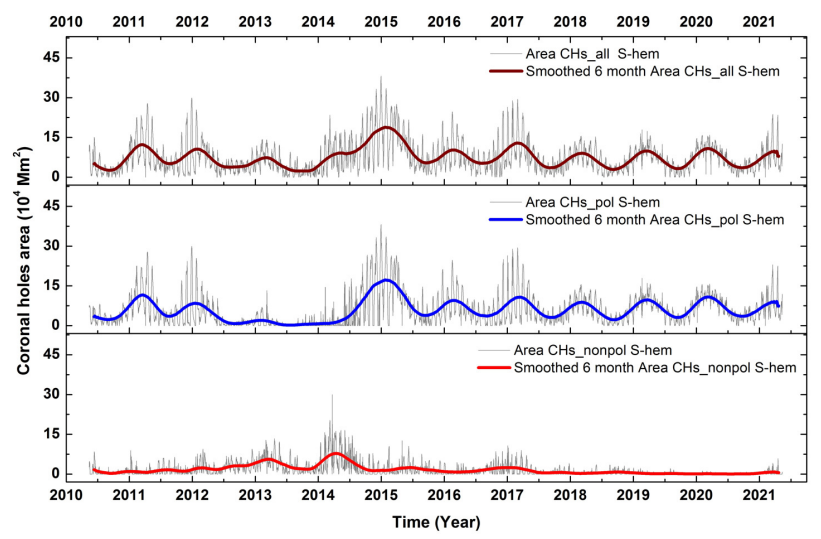

Figure 6. Dynamics of the areas of all, polar and non-polar $\mathrm{CHs}$ (from top to bottom) in the S-hemisphere in the period May 13, 2010 to May 13, 2021.

Figure 5 and Figure 6, from top to bottom, shows changes in the daily total area (gray lines) for all, polar and nonpolar $\mathrm{CHs}$ in the $\mathrm{N}$ and $\mathrm{S}$ hemispheres corresponding. The color curves are the CHs areas smoothed over 6 months.

The following can be noted upon analysis of the temporal distribution of polar and nonpolar CHs areas across hemispheres. In the N-hemisphere nonpolar CHs (20132014) (Figure 5 bottom plot) determine the coronal holes activity, because polar are very weak. They also determine the behavior of nonpolar holes for the entire disk (Figure 4, bottom plot). The similar situation we observe for polar CHs (2011, 2012 and 2015) of the S-hemisphere (Figure 6 and Figure 4, middle plot). Thus, it can be noted that in the period under study there was an asymmetry of the hemispheres in the localization of the maxima of the areas of polar and nonpolar CHs. Visible quasi-periodic oscillations on the middle plots Figure 5 and Figure 6 for Sch_pol are an artifact caused by the tilt of the sun's rotation axis during the year.

\subsection{Dynamics of sunspots area}

Data about daily sunspot areas taken from http:// solarcyclescience.com/AR_Database/daily_area.txt. Figure 7 show changes in the daily total sunspots area (gray lines) on the visible surface of the solar disk, $\mathrm{N}$ and $\mathrm{S}$ hemispheres (from top to bottom, respectively). The red curves are the sunspots area smoothed over 6 months. An asymmetry is observed in the distribution of the maximum of the sunspot areas. 2012 on the North, 2014 on the South.

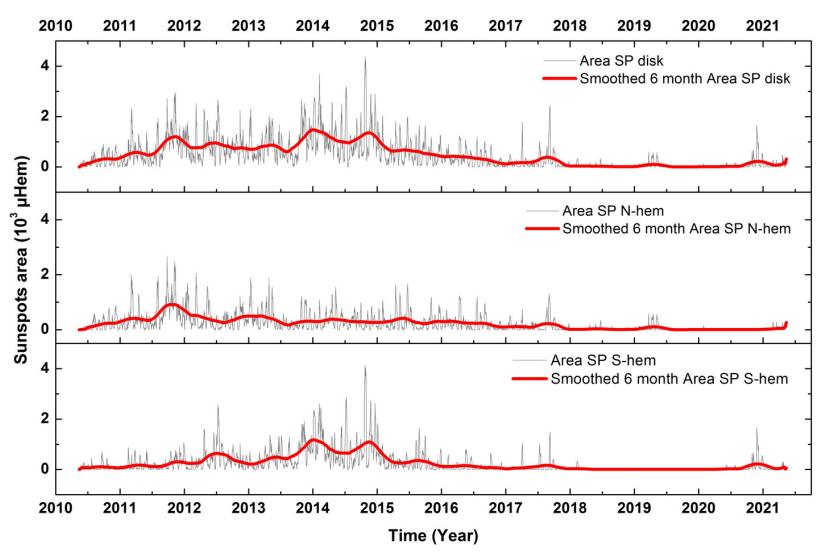

Figure 7. Dynamics of sunspots area on the visible surface of the solar disk, in $\mathrm{N}$ and $\mathrm{S}$ hemispheres (from top to bottom) in the period May 13, 2010 - May 13, 2021.

\subsection{Relationship between coronal holes and sunspots in hemispheres}

Investigating the $\mathrm{CHs}$ dynamics, we analyzed the relationship between the areas of the two types of CHs and sunspots in different phases of the solar cycle. We see the hemispheric asymmetry of the polar $\mathrm{CHs}$ and the sunspots areas (Figure 5 and Figure 7, middle plots; Figure 6 middle plots and Figure 7 bottom plots;). The daily total area of polar CHs increases at solar minima and decreases at the cycle maximum. This is consistent with the general concept of polar coronal holes as the main source of the solar dipole magnetic field. In both hemispheres (Figure 5 and Figure 6, bottom plots), there is a tendency to an increase in nonpolar $\mathrm{CHs}$ at the rasing phase and maxima solar activity (Figure 7 middle and bottom plots), to a decrease at the decay and minima phases. This confirms the assumption that nonpolar CHs are most likely associated with sunspots activity.

\section{Conclusion}

The dynamics of CHs during 11-year cycle was studied. Comparison of the variations in the daily total $\mathrm{CHs}$ areas with the sunspot areas made it possible to reveal some features of the dynamics of the polar and nonpolar $\mathrm{CHs}$ areas in the period May, 132010 - May, 13 2021. In the 24th solar activity cycle, the South led for polar CHs and the North led for nonpolar ones. There is a connection between the number and area of $\mathrm{CHs}$ and the phase of the solar cycle. The number and daily total area of polar CHs increases at the minima of solar activity and decreases at the maximum 
of the cycle. This is consistent with the general concept of polar CHs as the main source of the solar dipole magnetic field (e.g. Bilenko 2002; Sanderson et al. 2003; Wang 2009; Obridko and Shelting 2011; Fujiki et al. 2016; Lowder et al. 2017). An asymmetry in both the number and areas of polar coronal holes in the northern and southern hemispheres is observed. It is shown that the areas of nonpolar CHs change quasi-synchronously with sunspot activity, which suggests a physical connection between these two phenomena.

Acknowledgment: The SDO is part of the Living with a Star program of the National Aeronautics and Space Administration. The SDO/AIA data were provided by the Joint Science Operation Center (JSOC). We thank the Heliophysics Event Knowledgebase (HEK) project team for access to the CHs databases. We are grateful to the authors of http: //solarcyclescience.com/AR_Database/daily_area.txt for the opportunity to use sunspot area data. We thank the anonymous reviewer whose comments and critiques made it possible to improve the article.

Funding information: The authors state no funding involved.

Author contributions: All authors have accepted responsibility for the entire content of this manuscript and approved its submission.

Conflict of interest: The authors state no conflict of interest.

\section{References}

Andreeva O, Malashchuk V. 2020. Sunspot activity and coronal holes during the declining phase of cycle 24. Geomagn Aeron. 60(8):1093-1100.

Barra V, Delouille V, Kretzschmar M, Hochedez J. 2009. Fast and robust segmentation of solar EUV images: Algorithm and results for solar cycle 23. Astron Astrophys. 505(1):361-371.
Bilenko I. 2002. Coronal holes and the solar polar field reversal. Astron Astrophys. 396(2):657-666. https://doi.org/10.1051/ 0004-6361:20021412.

Cranmer S. 2009. Coronal Holes. Living Rev. Sol. Phys. 6: 3-66. https://doi.org/10.12942/lrsp-2009-3.

Dikpati M, Charbonneau P. 1999. A Babcock-Leighton Flux Transport Dynamo with Solar-like Differential Rotation. ApJ. 518(1):508-520.

Fujiki K, Tokumaru M, Hayashi K, Satonaka D, Hakamada K. 2016. Long-term trend of solar solar coronal hole distribution from 1975 to 2014. ApJ Lett. 827:L41. https://doi.org/10.1007/ s11207-011-9753-2

Hurlburt N, Cheung M, Schrijver C, Chang L, Freeland S, Green $\mathrm{S}$, et al. 2012. Heliophysics event knowledgebase for the Solar Dynamics Observatory (SDO) and beyond. Sol Phys. 275:67-78.

Karna N, Pesnell W, Zhang J. 2015. Appearances and statistics of coronal cavities during the ascending phase of solar cycle 24 . ApJ. 810(2):123-127. https://doi.org/10.1088/0004-637X/810/ 2/123.

Li K, Li Q, Gao P, Shi X. 2008. Cyclic behavior of solar full-disk activity. JGRA. 113(A11):A11108. https://doi.org/10.1029/ 2007JA012846.

Lowder C, Qiu J, Leamon R. 2017. Coronal holes and open magnetic flux over cycles 23 and 24. Sol Phys. 292:18. https://doi.org/ 10.1007/s11207-016-1041-8.

Obridko V, Shelting B. 2011. Relationship between the parameters of coronal holes and high-speed solar wind streams over an activity cycle. Sol Phys. 270:297-310, https://doi.org/10.1007/ s11207-011-9753-2.

Parker E. 1955. Hidromagnetic Dynamo Models. ApJ. 122:293-314. http://dx.doi.org/10.1086/146087.

Sanderson T, Appourchaux T, Hoeksema J, Harvey K. 2003. Observations of the Sun's magnetic field during the recent solar maximum. J Geophys Res. 108(A1):1035. https://doi.org/10. 1029/2002JA009388.

Shimojo M. 2013. Unusual Migration of Prominence Activities in the Southern Hemisphere during Cycles 23-24. PASJ. 65:S16.

Verbeeck C, Delouille V, Mampaey B, De Visscher R. 2014. The SPoCA-suite: Software for extraction, characterization, and tracking of active regions and coronal holes on EUV images. Astron Astrophys. 561:A29.

Wang Y. 2009. Coronal holes and open magnetic flux. Space Sci Rev. 144:383-399. 